\title{
Improving Particle Segmentation from Process Images with Wiener Filtering
}

\author{
Lauri Laaksonen, Nataliya Strokina, Tuomas Eerola, \\ Lasse Lensu, and Heikki Kälviäinen \\ Machine Vision and Pattern Recognition Laboratory (MVPR) \\ Department of Information Technology \\ Lappeenranta University of Technology (LUT) \\ P.O. Box 20, FI-53851 Lappeenranta, Finland \\ firstname.lastname@lut.fi \\ http://www2.it.lut.fi/mvpr
}

\begin{abstract}
While there is growing interest in in-line measurements of paper making processes, the factory environment often restricts the acquisition of images. The in-line imaging of pulp suspension is often difficult due to constraints to camera and light positioning, resulting in images with uneven illumination and motion blur. This article presents an algorithm for segmenting fibers from suspension images and studies the performance of Wiener filtering in improving the sub-optimal images. Methods are presented for estimating the point spread function and noise-to-signal ratio for constructing the Wiener filter. It is shown that increasing the sharpness of the image improves the performance of the presented segmentation method.
\end{abstract}

Keywords: pulp suspension, fiber segmentation, Wiener filtering, machine vision, image processing and analysis.

\section{Introduction}

The paper industry has recently shown increasing interest in the in-line measurements as they could provide information on the state of the process on-line, making it possible to control the process while the product is still forming. Reliable on-line estimates could be used for process optimization, automation and avoiding breaks and delays in the process. However, accurate on-line measurements are difficult to obtain due to restrictions to camera and illumination, low contrast of many of the measured particles and the execution times required for timely measurements.

There are methods for obtaining certain measures related to the papermaking process and the quality of the end-product. Sithole and Filion compare quality measurements of recycled pulp [12. Wang and Hubbe 13 propose a method for measuring the electrical properties of fiber surfaces [13] and Saarela et al. [11] use a streak camera for measuring the fines content of pulp with unknown consistency. These methods, however, tend to be specific for a certain measurement and are often performed on pulp sheets in laboratory conditions.

A. Heyden and F. Kahl (Eds.): SCIA 2011, LNCS 6688, pp. 285 294, 2011.

(C) Springer-Verlag Berlin Heidelberg 2011 
Among the most important factors affecting the quality of the end product are the properties and formation of the fiber web. The characteristics of the fibers have a significant effect on the quality of the pulp and the end product. For example, the length and coarseness of fibers affect the flocculation (the forming of mass concentrations within the suspension) and the mobility of the fibers, both of which are factors in the uniformity of the suspension and the extent the suspension uniformity can be altered. [10

Before it is possible to make measurements of the fiber characteristics, the fibers need to be segmented from the suspension images. The low contrast and varying intensity values of the pixels depicting fibers cause thresholding and traditional edge detection methods to perform poorly.

This article presents an approach for fiber segmentation from pulp suspension images, which is a part of a fully automated system of pulp flow analysis for the inline measurements. Wiener filtering for image restoration is presented. The estimation of the Wiener filter parameters is discussed. An algorithm for segmenting fibers is proposed and its feasibility as a part of on-line measurements is studied. Experiments concerning the effect of the Wiener filtering on the performance of the segmentation algorithm are presented and discussed.

\section{Imaging and Image Processing}

The images of flowing low-consistency pulp used in this article were taken from within a pilot process in LUT FiberLaboratory in Savonlinna. The camera used was a Guppy F-046B, manufactured by Allied Vision Technologies. The $8.3 \mu \mathrm{m}$ $\times 8.3 \mu \mathrm{m}$ CCD-cells of the camera could produce a maximum of $780 \times 582$ pixel images with a framerate up to 49.4 frames per second [1]. As the in-line environment of papermaking process offers limited or no accessibility for a camera with a normal lens, the camera was fitted to a Richard Wolf borescope with a video lense from the same manufacturer.

To improve the contrast of the fibers, they were processed with an agent that radiated fluorescent light. One of the pulp images for testing the fiber segmentation algorithm is presented in Fig. 4.

\subsection{Wiener Filtering}

Wiener filtering is an inverse filtering method that takes the statistical characteristics of image noise into account. Both the image and image noise are considered as random variables and the undistorted image $f$ is estimated by minimizing the mean square error between the undistorted image and estimated image. 5]

The error measure for the mean square error is calculated from

$$
e^{2}=E\left\{(f-\tilde{f})^{2}\right\},
$$

where $e^{2}$ is the squared error, $\tilde{f}$ the estimate of the undistorted image and $E\{\cdot\}$ is the expected value of the argument [5]. The degraded image is defined as 
$g=h(f+N)$, where $h$ is the point spread function and $N$ the additive noise in the image.

The noise-to-signal ratio (NSR) required for the filtering is rarely known, but it can be approximated by a constant 9. Replacing NSR with constant $\gamma$, the frequency representation of $\tilde{f}$ minimizing the error function in Eq. 1 is gained from

$$
\tilde{F}(u, v)=\left[\frac{1}{H(u, v)} \frac{|H(u, v)|^{2}}{|H(u, v)|^{2}+\gamma}\right] G(u, v),
$$

where $H$ is the frequency domain representation of the point spread function of the whole system and $G$ the frequency domain representation of the degraded image. 5] The parameters required for the filtering are now reduced to point spread function $H$ and the constant estimating the noise-to-signal ratio $\gamma$.

Estimation of point spread function. The point spread function of a camera can be estimated from images with a sharp edge 8 . The location of the edges present in an image is derived by performing Canny edge detection [3] and performing connected component analysis on the found edges.

To find a proper estimate for the edge, RANSAC (Random Sample Concensus) [4] is used to find the line represented by the edge pixels. A number of edge profiles are taken along the line, five pixels from both sides of the edge. The mean edge profile is calculated and normalized between [ $\left[\begin{array}{ll}0 & 1\end{array}\right]$ by

$$
Z_{i}=\frac{p_{i}-p_{\min }}{p_{\max }-p_{\min }}
$$

where $p_{i}$ is the $\mathrm{i}$ :th intensity value of the mean edge profile, $p_{\max }$ is the maximum value of the mean profile, $p_{\min }$ the minimum and $Z_{i}$ the normalized profile value.

The normalized values are compared to an ideal, infinitely sharp edge $p_{\text {ideal }}$, positioned at the center of the normalized profile. The ideal edge depicts an instant transition between the edge and non-edge pixels. In practice, the ideal edge cannot be achieved for normal or high-resolution cameras as it would require alignment of the edge target and the camera CCD-cells, and perfect illumination conditions. Thus, this method of estimation always yields some amount of point spread.

The directional point spread estimates $e_{x}$ and $e_{y}$ are calculated as $\left|p_{x}-p_{\text {ideal }}\right|$ and $\left|p_{y}-p_{\text {ideal }}\right|$, respectively. The point spread function matrix $M_{p s f}$ is calculated from the column vectors $e_{x}$ and $e_{y}$ by

$$
M_{p s f}=\frac{e_{y}^{T} \times e_{x}}{\sum\left(e_{y}^{T} \times e_{x}\right)} .
$$

Estimation of noise-to-signal ratio. Murphy et al. 7] present two suitable methods to estimate the noise in an image. Taking a large uniform area of the image, the noise can be measured as the standard deviation of the intensity values of the area. Another approach is to take two images of the same target, subtract one image from the other and calculating the standard deviation of the 
difference. This method can be improved by increasing the number of images taken and subtracting the mean image from each of the original ones.

With the statistical characteristics of the noise known, an estimation of the mean noise-to-signal ratio (NSR) can be calculated for the images from

$$
N S R=\frac{\sigma(n)}{\sigma(u)},
$$

where $\sigma(u)$ is the standard deviation of the undistorted image (i.e. the mean image of the set) and $\sigma(n)$ is the standard deviation of the noise [2].

As the environmental conditions are expected to remain constant for the duration of image acquisition and the noise is assumed to be caused mainly by characteristics of the camera, the NSR is expected to remain approximately the same regardless of the imaged target. However, as temperature and other environmental factors may change over time in the process environment, re-estimation of the noise model may be required from time to time.

Estimation result. The sharp edges for estimating the PSF were produced in the image with two thin razors imaged from the distance of $380 \mathrm{~mm}$ (see Fig. 1). It was assumed that the thin edges can be accurately represented by straight lines to the accuracy of $780 \times 582$ pixels captured by the camera. Fig. 2 shows the estimated edge profiles. A visual representation of the PSF estimated from the given images is shown in Fig. 3 .

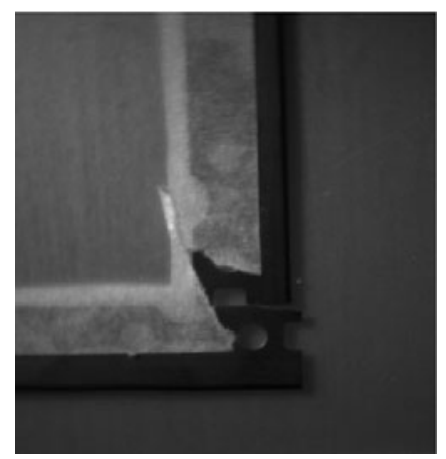

Fig. 1. Image for estimating the edge spread function

The noise caused by the camera, measured over ten images, was normally distributed (see Fig. 3) with zero mean and standard deviation of 0.0032 . The Lilliefors test [6], a statistic test for determining if data is normally distributed when the mean and variance must be estimated from the sample, was performed on the noise model of each image. The test suggested that the models are normally distributed with the probability of $99.9 \%$. 


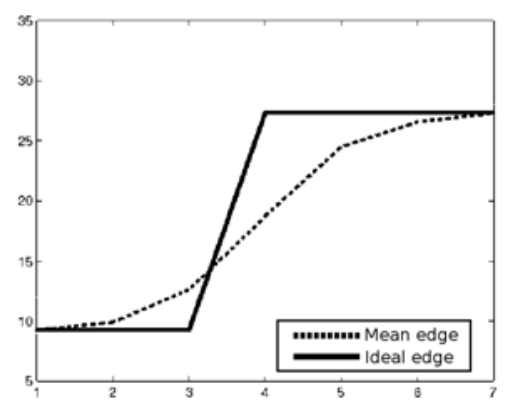

(a)

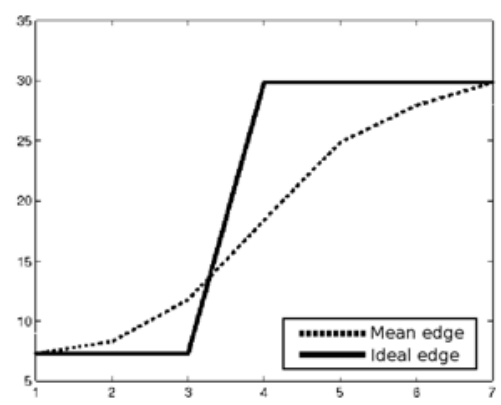

(b)

Fig. 2. Mean edge profiles: (a) Horizontal; (b) Vertical

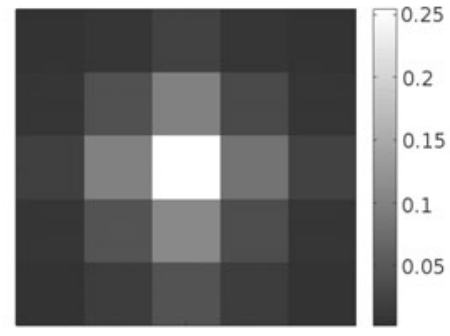

(a)

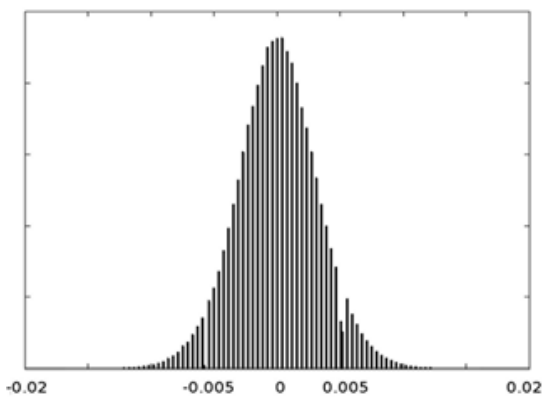

(b)

Fig. 3. Camera noise: (a) Visual presentation of the PSF; (b) Distribution of the noise.

\subsection{Fiber Segmentation}

The approach for detecting the fibers is summarized in Algorithm 1. Due to poor performance of standard edge detection filters, the initial segmentation of fibers is done by a set of filters designed specifically for the images in Fig. 4 . The filter assumes the fibers to manifest as long, thin objects in the images, with intensity higher than the background. While there is a loss of generality due to the resolution dependency of the filter set, the designed filters supercede the traditional edge detection filters in performance.

The filter set is generated by rotating the filter

$$
M=\left[\begin{array}{lllllllll}
-2 & -1 & 0 & 1 & 4 & 1 & 0 & -1 & -2 \\
-2 & -1 & 0 & 1 & 4 & 1 & 0 & -1 & -2 \\
-2 & -1 & 0 & 1 & 4 & 1 & 0 & -1 & -2
\end{array}\right]
$$

by $45^{\circ}$ both clockwise and counter-clockwise to produce the filters for diagonally positioned fibers, and by rotating $M$ by $90^{\circ}$ for detecting horizontal fibers. 


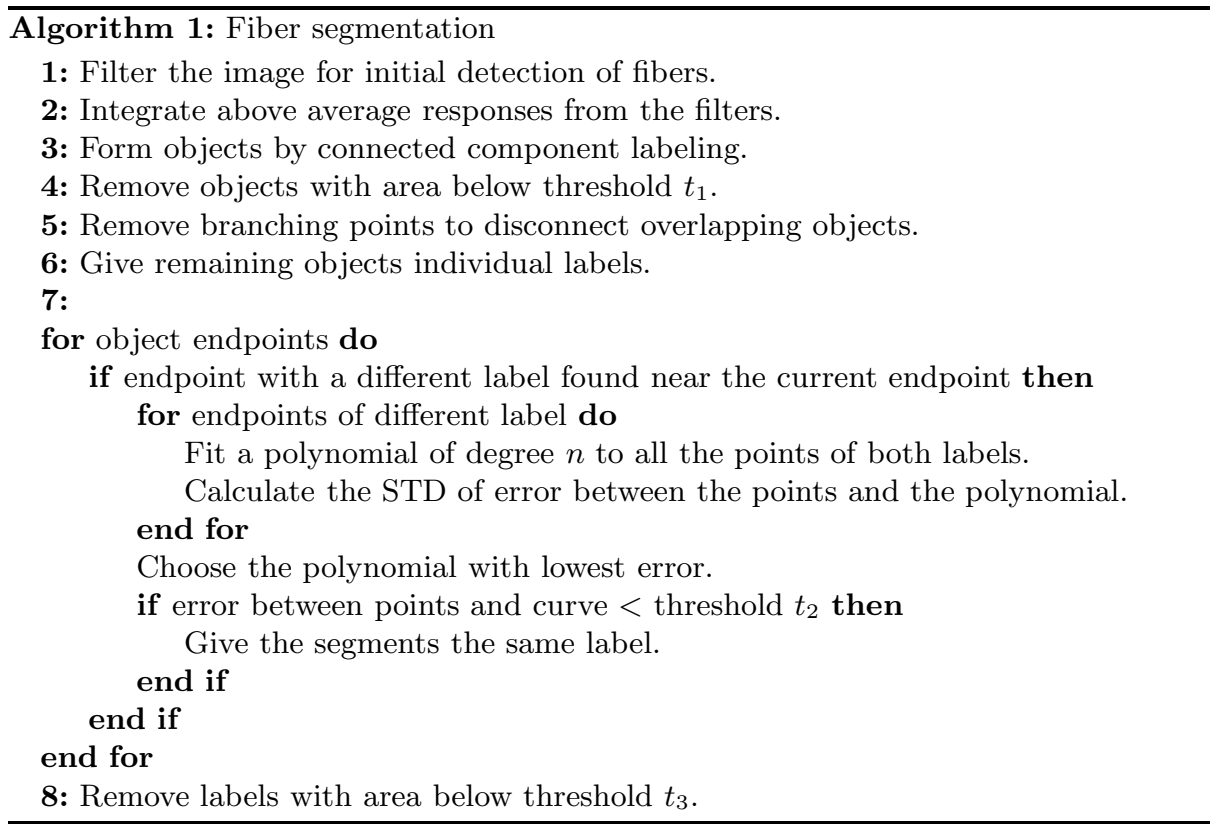

Before integrating the filter responses, any response below the mean intensity of the image is removed to reduce false responses. Connected component analysis is performed on the remaining responses to form objects depicting the fiber segments. Objects with area below $t_{1}$ are removed as noise (Step 4).

Binary thinning, i.e. the iterative removal of the outer edge pixels of an object, is performed in Step 5. The segments with a width of single pixel are disconnected by removing any branch point on the single pixel wide path of a fiber segment. This results in a number of objects with an area of only one or few pixels. These objects are considered as noise and removed, while the remaining segments are given individual labels.

The area around the endpoints of each labeled segment is investigated. If an endpoint with a different label is found, a polynomial curve of degree $n$ is fit to all pixels of both labels. The standard deviation of the error between the datapoints and the fitted curve is calculated. If the error is below a threshold $t_{2}$ the segments are considered to belong to the same fiber and are given the same label.

In the case of multiple nearby labels, the curve is fit to the segment containing the investigated endpoint and to the pixels of all the labels in turn, and the curve between the label with least error in the fit is tested against the threshold. Objects with pixel area remaining below $t_{3}$ after connecting the segments are removed. The segmented fibers, can be seen in Figures 5 . 


\section{$3 \quad$ Experiments and Discussions}

The presented segmentation method was applied to the set of 6 images, one of which is presented in Fig. [4 Using the first image the following parameters of the algorithm were defined: $t_{1}=40, t_{2}=5, t_{3}=30$ and $n=3$. The method correctly classified reasonably difficult cases. In Fig. 6d a closer look is taken at several typical situations the method faces when deciding if segments close to each other should be classified as one fiber.

Fig. 6a shows a simple case of joining the segments of a straight fiber. In Fig. 6b the mean interpolation error of the curve fit to the pixels of the two long segments is too large and the segments are classified as separate fibers. A more difficult case is shown in Fig. 6c where the segments of the two straight fibers are connected, while the two fibers are classified as separate and the segments of one fiber are not classified as a part of the other. One of the most difficult cases is shown in Fig. 6d, where a straight fiber in several segments is overlapped by a curved fiber in two segments. For this instance, the method correctly classifies the overlapping fibers.

The effect of edge sharpening by the Wiener filter on the performance of the fiber segmentation method was tested on the same set of six suspension images. The output of the segmentation algorithm was referenced to a visual estimation of the correct fibers in the image. While the visual estimation was done by a non-expert, the fibers in the used pulp images are salient enough to assume a reasonable accuracy for the estimation.

The results of the segmentation were divided in to three categories: detected fibers (det.) with most of the fiber segments were found and correctly labeled, partly detected fibers (par.) with a part of the fiber detected, but missing segments, and incorrect (inc.) with wrong segments joined or noise segmented as fiber segment. The number of fibers (num.) was manually estimated for each image to provide ground truth for the segmentation result.

Filtering the image with Wiener filter resulted in an increase of detected segments in all categories. The amount of complete fibers detected was significantly increased along with the amount of the more obscure fibers partially found. While there was some increase in the false filter responses detected as fiber segments, most of the final false segments were relatively small and could be removed by pixel area based thresholding, although at the cost of small true segments.

The results of the segmentation are shown in Tab. 1. The Matlab implementation of the segmentation algorithm was tested on a desktop computer with two Intel Pentium 3.00 GHz processors and 2.0 GB of memory. On the average, the method performed the segmentation for the unfiltered images in approximately 0.56 seconds. When performing the algorithm on filtered images the segmentation took approximately 0.88 seconds due to increased number of detections (both real segments and noise). 
Fig. 4. An example of the test images for the fiber segmentation algorithm

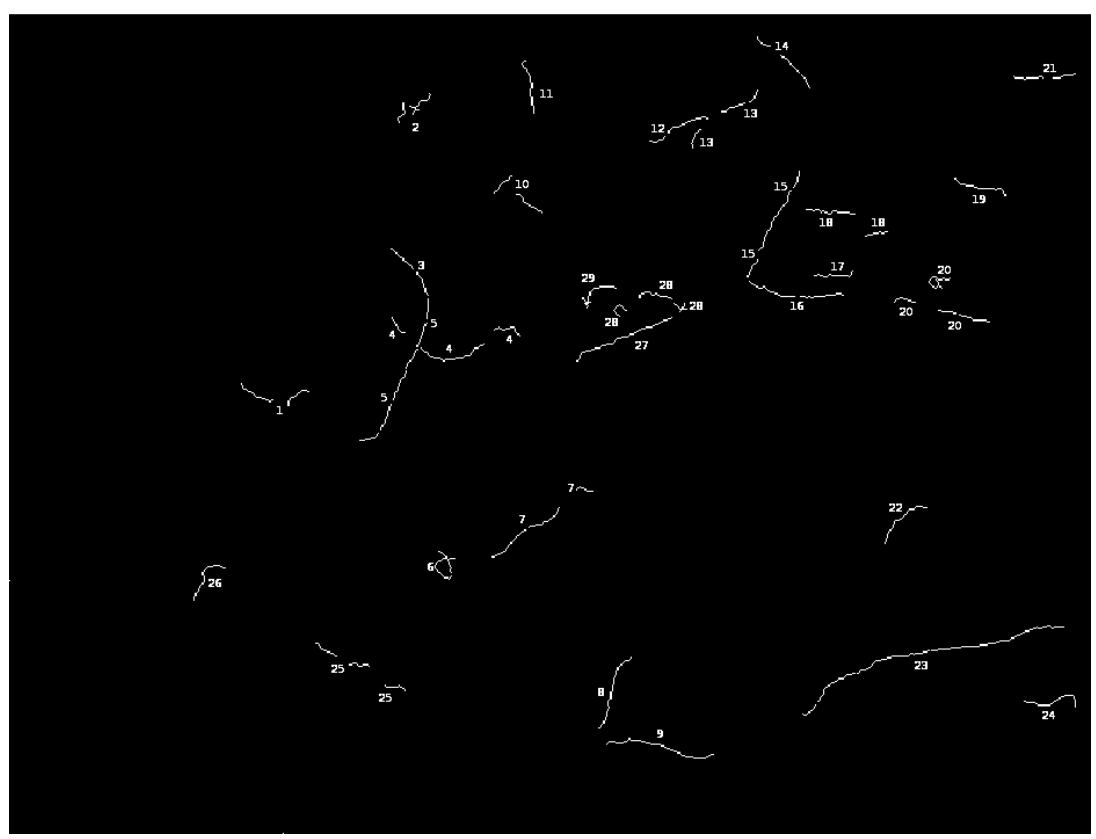

Fig. 5. Segmented fibers with small segments removed 


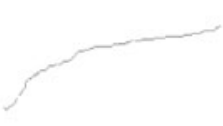

(a)

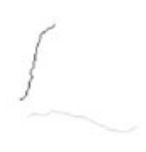

(b)

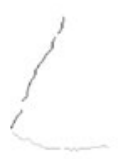

(c)

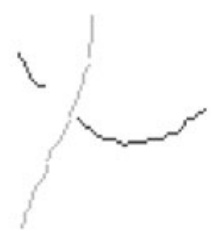

(d)

Fig. 6. Closer examination of segment joining results: (a) Segments recognized as a straight fiber; (b) Segments recognized as separate fibers; (c) Segments recognized as two separate straight fibers; (d) Segments from overlapping fibers recognized as a straight and a curved fiber

Table 1. Effect of Wiener filtering on fiber segmentation. (det.) correctly detected, (par.) partially detected, (inc.) noise segmented or segments joined incorrectly, (num.) estimated number of fibers.

\begin{tabular}{l|ccc|ccc|c} 
& \multicolumn{3}{|c|}{ Filtered image } & \multicolumn{3}{c|}{ No filtering } & \\
\hline & \multicolumn{3}{|c}{ det. par. } & inc. & det. & par. inc. & num. \\
Image 1 & 9 & 7 & 5 & 4 & 9 & 0 & 19 \\
Image 2 & 6 & 1 & 4 & 4 & 0 & 2 & 11 \\
Image 3 & 7 & 4 & 4 & 3 & 5 & 0 & 16 \\
Image 4 & 2 & 5 & 1 & 0 & 2 & 0 & 12 \\
Image 5 & 2 & 4 & 1 & 2 & 1 & 0 & 14 \\
Image 6 & 1 & 6 & 1 & 0 & 1 & 0 & 9
\end{tabular}

\subsection{Future Work}

The segmentation of the fibers is the first step in in-line measurements. There is still demand for tracking the fibers to determine the flow, classification of the fibers and further study of the process based on the measurements. Methods for studying the formation and behaviour of the fiber web are also of interest.

\section{Conclusion}

While the in-line measurements of paper making process are difficult due to restrictions to the location of the camera and positioning of the light, the benefits are significant. In this work, a method for segmenting fibers from pulp suspension images was presented, along with methods for estimating the point spread function and the noise-to-signal ratio for constructing the Wiener filter. The improved sharpness of the image after Wiener filtering significantly improved the performance of the fiber segmentation algorithm. 


\section{Acknowledgements}

The research was carried out in the "PulpVision" project (TEKES project 70010/10) funded by the European Union and the participating companies. The authors wish to acknowledge the FiberLaboratory in LUT for cooperation and providing the pulp images used in the article.

\section{References}

1. Allied Vision Technologies. Guppy Techical Manual (2009)

2. Buades, A., Coll, B., Morel, J.M.: A review of image denoising algorithms, with a new one. Multiscale Modeling and Simulation 4, 490-530 (2005)

3. Canny, J.: A computational approach to edge detection. IEEE Transactions of Pattern Analysis and Machine Intelligence 8, 679-698 (1986)

4. Fischler, M.A., Bolles, R.C.: Random sample consensus: a paradigm for model fitting with applications to image analysis and automated cartography. Communications of the ACM 24, 381-395 (1981)

5. Gonzales, R.C., Woods, R.E.: Digital Image Processing, 3rd edn. Pearson Prentice Hall, London (2008)

6. Lilliefors, H.W.: On the kolmogorov-smirnov test for normality with mean and variance unknown. Journal of the American Statistical Association 62, 399-402 (1967)

7. Murphy, B.W., Carson, P.L., Ellis, J.H., Zhang, Y.T., Hyde, R.J., Chenevert, T.L.: Signal-to-noise measures for magnetic resonance imagers. Magnetic Resonance Imaging 2, 425-428 (1993)

8. Szeliski, R., Joshi, N., Kriegman, D.J.: Psf estimation using sharp edge prediction. In: IEEE Conference on Computer Vision and Pattern Recognition, CVPR (2008)

9. Pang, M.-C.: A novel blind super-resolution technique based on the improved poisson maximum a posteriori algorithm. International Journal of Imaging Systems and Technology 12, 239-246 (2002)

10. Robertson, G., Olson, J., Allen, P., Chan, B., Seth, R.: Measurement of fiber length, coarseness, and shape with the fiber quality analyzer. Tappi Journal 82, 93-98 (1999)

11. Saarela, J., Törmänen, M., Myllylä, R.: Measuring pulp consistency and fines content with a streak camera. Measurement Science and Technology 14, 1801-1806 (2003)

12. Sitholé, B., Filion, D.: Assessment of methods for the measurement of macrostickies in recycled pulps. Progress in Paper Recycling 17 (2008)

13. Wang, F., Hubbe, M.: Development and evaluation of an automated streaming potential measurement device. Colloids and Surfaces A: Physicochemical and Engineering Aspects 194, 221-232 (2001) 